\title{
Material-Based Design: Improving a Continuous Changing Praxis
}

XXIV International Conference

of the Iberoamerican Society

of Digital Graphics

Medellin | Colombia

\author{
Denise Mônaco dos Santos \\ Universidade Federal de Viçosa | Brazil | denise.monaco@ufv.br \\ Ingrid Paoletti \\ Politecnico di Milano | Italy | indrid.paoletti@polimi.it
}

\begin{abstract}
Material-based design has become a wide approach, gathering under the same umbrella different design practices, which deserve to be accurately reexamined. Material-based design involves material performance in complex integration with informational data and processes. However, material-based design operates a new material condition with historical, social, ethical, and political effects. A new term can be used for materiality not only as an objective substance but also as a knowledge-based interrelated element that requires a balance among actors, tools, matters, and meaning.
\end{abstract}

Keywords: Material-based design; Digital design theory; Digital design practices; Materials systems; New materials.

\section{INTRODUCTION}

There are different ways that material information can perform in digital design processes and act as a material design approach for architects, engineers, and designers. However, are we really identifying them when we use the terms 'digital materiality', 'material computation', or 'digital tectonics'?

Material-based design has become a wide approach, gathering under the same umbrella different design practices, which deserve to be accurately reexamined. Topics, such as, the emergence of 'materials by design', the potential increase in performative transformation of materials, the capacity to produce adaptive and dynamic materials systems, further reassert the material as a design active element. The use of large databases, simulation, and optimization, provide us with choices of creating nonconventional materials and process innovation.

The principal contribution of this study is to reveal the transformative scenarios of the prominent role of the material in digital design, potentially contributing to improving the praxis in the context of continuous change. A new term can be used for materiality not only as an objective substance but also as a knowledge-based interrelated element that requires a balance between actors, tools, matters, and especially meaning.

The interest that first stimulated this study was to point to the particularities of digital design processes driven by materials. It started from the assumption that the term material-based design does not define with exclusivity and accuracy the current stage of digital design centered on materials and their processes. Initially, this definition focused on the more substantial changes and differences between traditional processes and informed tectonics, which constitutes the general digital design, involving or not in-depth research on materials (Oxman, 2012; Oxman, 2017).
In other words, the use of the term seems to be imprecise: material-based design would also define processes in which there is not necessarily an evident manipulation of materials at the early stages of design or an "ability to design materials in a bottom-up manner" (Bechthold \& Weaver, 2017, p.1). The material would not be the starting point of the design process. The indistinct and imprecise use of the term sometimes implies 'material innovation' in what would be the current procedure of digital processes, without being innovative in this field. However, more than imprecisions, we believe that there is a complex diversity of processes in a supposed singularity.

The correct nomination of a process is important, but it is a theoretical discussion. How does it assist digital design practices? It gives support to the challenges that design driven by materials requires: transdisciplinary knowledge in action, which can impose a new modus operandi. These are recent experiences constantly updated and induced by technical and technological requirements, which, consequently, encourage architects to work not just with their usual peers and disciplines.

To support the problematizations and discussions of this investigation, it followed two main steps: (i) a literature review, exploring a limited sample of research in the fields of digital design and material culture; and (ii) the elaboration of an implementable definition for materialbalanced design. To illustrate, this discussion brings up the experiences and studies carried out at two research centers, one European and one South American.

\section{THE PROMINENCE OF MATERIALS IN DIGITAL DESIGN}

\section{INFORMED TECTONICS}

Digital design a well-defined field in architecture, implying an intrinsic relationship between design and the use of a specific set of digital tools for design itself and for manufacture/fabrication/production (Carpo, 2013; Carpo, 
2017; Oxman \& Oxman, 2014; Oxman, 2017; Caetano \& Leitão, 2020). It is a highly informed design process associated to the use of different spheres of computability, from an instrumental toolset, which allows form generation and varying levels of simulation, analysis, and optimization linked to the production of prototypes of different scales. The design thinking emphasis is procedural and nonsubjective.

In this context, the intrinsic relationships among form, structure, and explicitly informed material properties constitute particulars. Informed tectonics characterizes digital design processes whose tectonics is computationally informed through integration of data on materials, structure, and production processes, defined at the early stages of project development (Oxman, 2012). According to the author, the conventional order of the tectonic constitution of architecture has been altered in digital design processes, which are not only subject to the sequential model of 'form-structure-material' but also encompass other arrangements, e.g., 'material-structureform', characteristic of material-based processes (Oxman, 2017).

\section{INFORMED MATERIALS}

In the development of digital design theory, the interaction between materials data and digital design processes began to be highlighted and examined in depth from the early 2000's. According to Yazici and Tanacan (2020) "the use of $C D$ [computational design] in architectural design processes has extended the meaning of digital materiality as identified almost a decade ago, thus making the role of materials even more important today" (para. 2).

In this specific context, it seems difficult to point to seminal theoretical studies or to track periodizations, as in studies addressing the very recent history of digital design theory (Carpo, 2013; Oxman \& Oxman, 2014; Caetano \& Leitão, 2020). Even so, some theoretical discussions are noteworthy, not only because they reflect on the material attributes engendered by digital design in different ways and at various stages of the process, but precisely because they are closely associated with practical experiments. Neri Oxman (2010) uses the term material-based design computation to define "a set of computational strategies that support the integration of form, material and structure, incorporating physical strategies to find shapes with analysis and digital fabrication" (p.5), regarding this praxis as a new field. For the author, this field "is based on the premise that material, structure, and form can become inseparable entities of the design process, which relate to matter, performance and geometry respectively" (N. Oxman, 2010, p.148); a synergetic approach that integrates material organization and behavior into digital tools. The close involvement of the material attributes with the design process is also behind the term digital materiality created by Gramazio and Kohler (2008). Researchers with extensive practical exploration, the authors highlight "the integration of decisions relevant to manufacturing in coded projects allows the architect to control complex interactions between singular material elements and facilitates the direct generation of machining data" (Gramazio, Kohler \& Oesterle, 2010, p.111); therefore, they conceptually direct the ambivalent fusion of the terms digital and material, bringing together two constitutively distinct and even opposing entities, "as the synthesis of two apparently distinct worlds" (Yazici \& Tanacan, 2018, p.68).

The material-based process agenda, established over the past twenty years, clearly indicates the possibility of architecture encompassing materials by design, e.g., smart materials, responsive materials, living materials, and materials engineered for specific use-qualities. The materials themselves subject to design can be nonhomogeneous, non-standardized, non-stable, and/or noninert, involving innovative production methods. It is the active matter, an agenda associated with the transformations in material science that produce new materials, experiencing its limits, modifications, and mixtures. According to Bechthold and Weaver (2017), the combination of this trend with "the advent of technologies such as digital manufacturing, robotics and 3D printing not only accelerated the development of new construction solutions, but also led to a renewed interest in materials as a catalyst for new architectural projects" (p.1). According to the authors, the design of new material systems and their applications emphasizes interdisciplinarity and requires collaboration among architects, designers, engineers, and material scientists, among other professionals. It is a context in which the complexity of materials prevails, which, in addition to raw materials, natural or synthetic, involves the mix of matters or substances and different production processes. Materials are also products themselves.

\section{SINGULARITY AND COMPLEXITY}

Proceedings of annual international scientific events have been important allies in the disseminating of recent scientific research and practical academic studies in the field of digital design. In this context, there stand out the sibling associations ACADIA, CAADRIA, eCAADe, SIGraDi, ASCAAD, and CAAD Futures, which keep their annals' congresses public on the open platform CumInCAD - Cumulative Index about publications in Computer Aided Architectural Design. According to Caetano and Leitão (2020), these events promote debates about theoretical and practical issues, indicating the latest practices, emerging tools and techniques, and ordinary needs.

Materials have not yet constituted exclusive themes in congresses of the aforementioned associations, although those from ACADIA have dedicated specific topics to studies with this focus for over fifteen years, and those from eCAADe for almost ten years. Since 2015 eCAADe indicates the submission of papers under the topic Material studies, wherein 2016 the topic was named Fabrication \& Material Studies and in 2019 Material studies and innovation. At the 2020 eCAADe, the term material is presented as part of the topic Health and materials in architecture and cities. ACADIA provided the following topics: Material science (biomaterials, computational materials, etc.) and Geomatics (composite materials and smart assemblies) in 2015, Programmable materials material agency in 2016; Challenges/disruptions in the material and construction front in 2017, Materializations of computational design strategies and Composite materials and smart assemblies in 2018, Composite materials and tectonics in 2019, and Material processes in 2020. The other sibling associations SIGraDi and ASCAAD have dedicated for the first time an exclusive topic to materials at their 2020 events, Sustainable materials and manufacturing processes and Programmable and smart 
materials, respectively. At the 2019 SIGraDi event, held together with eCAADe, the topic of Material studies and innovation was adopted by both (ACADIA, n.d.; ASCAAD, n.d.; CAADRIA, n.d., eCAADe, n.d.; SIGraDi, n.d.).

The emphasis on materials-related topics within the wider scope of digital design has begun to emerge, not coincidentally, at these events almost at the same time as in other scientific and academic circles. Even so, two issues can be highlighted: the diversity in naming and featuring topics of this nature and the time delay geographically circumscribed to areas besides Europe and North America. It is possible to situate the issue of terminological diversity in material-based design within the digital design development context itself, whose specializations, from the procedural perspective, continually increase, and occurs simultaneously with curation and dissemination of research and studies (Caetano \& Leitão, 2020). More importantly, then, it is to indicate that this diversity of terms directly corresponds to the diversity of processes.

Tectonics, with regard to digital design, associates material entities with formal (or geometric features of the form) and structural aspects. In this sense, the material is the tangible component of tectonics, the medium that gives physicality to structure and form. However, in some situations in material-based design, the material is a support whereas in others an end in itself. In any of these cases, however, the material is always in a relationship. It is a set of intangible data incorporated actively to a process, constantly being updated, integrated into a larger and more complex data network, which also includes the prospect of becoming tangible. By means of current computational resources, this data integration takes place at very deep levels, whether we are dealing with design situations that seek to improve material properties, such as those situations in which new materials are created, or even new material systems. In both cases, however, the objective is always to establish a specific performance: material performance. We understand material-based design as any and al processes in digital design that involve material performance.

Isolating the material component in digital design, with its complex integration of informational data, is difficult. In this context, the limits of categorizations and taxonomies, which seek to determine their character and features, are presented. This task begins by explaining conceptually the terms that name them, and much has been done in this direction (Oxman, 2012; Oxman, 2017; Yazici \& Tanacan, 2020; Caetano \& Leitão, 2020). However, at a deeper level, one comes up against the deeper operational elements of the tools in action, the processing of data at the algorithmic level. In this sense, the singularization of material-based design processes in architecture should seek to elucidate: How does materials data integration take place? How integrated can these data be so that the horizon of materialization is not lost? What are all actors involved, human and non-human, e.g., tools and devices? Which skills should be addressed by the disciplines? And still, what areas of knowledge can be added?

\section{PERFORMATIVE DESIGN THINKING}

We assume that material-based design, materia computation (Menges, 2012), digital materiality, and material - tectonics are different conceptualizations for the same approach to digital design in which material data is prominent. It operates mainly at the level of materials but does not dissociate them from other digital design components. It is a complex integration of material data into a set of associated tools of form generation, simulation, analysis, optimization, and fabrication. Despite being multifaceted, all operate at a deeper level of performative design thinking, associated to environmental sustainability. Its objectives comprise the search for solutions to lower consumption of matter and raw material and to extend the lifespan of materials, the development of materials with recyclable properties, low energy consumption in manufacturing, assembling, disassembling, and discarding components.

The current stage of material-based design is one of constant improvement, exchange and transference of practices and knowledge, and consolidation of a modus operandi guided by both performative design thinking and the imperative of technical and technological development.

\section{PERFORMATIVE DESIGN PRACTICES}

Despite the geographically circumscribed delay mentioned above, material-based design practices are increasingly consolidated and disseminated throughout the world. Although one cannot ignore the socioeconomic inequalities that are manifest territorially in the scientific and technological fields, the possibilities of the informational economy support the diffusion and exchange of information and knowledge in a spatially decentralized logic.

The aim of presenting the research on material-based design below, carried out at Nó.Lab (Universidade Federal de Viçosa, Brazil) and Material Balance Research Lab (Politecnico di Milano, Italy), is to illustrate features of this specialty in digital design. Although these groups and studies have different backgrounds, both practical experiments focus on the attributes of materials and on the relationships that integrate material, performance, and manufacturing. Both studies are representative of current practical experiments carried out in academia that foster material-based processes as part of architecture instruction and research.

Research 1 (Figure 1) focuses on the measurement of the mechanical behavior of materials, specifically the evaluation of the mechanical behavior of ABS and PLA polymers in the form of thermoplastic filaments commonly used in affordable 3D printers, subjected to axial compression and flexion forces (Martinez et al., 2019). It is the first stage of an ongoing research project, the first tests of which have indicated different compressive strengths depending on the internal mesh, performance, and dimensional deformation of the materials under investigation. Later, other international normative methods for mechanical testing of plastics and polymers, used in some simulation plugins, will be used with low-cost printers to create simulation environments that approximate postprint reality. It is a research driven by material structural performance.

Research 2 (Figure 2) also uses additive manufacturing technologies, albeit aimed at creating a constructive component of complex geometry, "a Triply Periodic Minimal Surfaces as the solution for the adaptive lattice structure" 
(Behforouz \& Pauletti, 2019). It employs a light-weight and porous structural component that can hold mycelium composite. "Mycelium composite is a promising alternative material in terms of thermal and acoustic as well as fire safety performance. It exhibits an exceptional end-of-life stage, replenishing the soil as it completely composts after disposal. However, it is suggested that mycelium composite still does not qualify as a structural material" (Behforouz \& Pauletti, 2019, para.1). This research mainly addresses performance environmental issues centered in Circular Economy, Cradle to Cradle, Upcycle, Biofabrication as opposed to conventional construction methods.

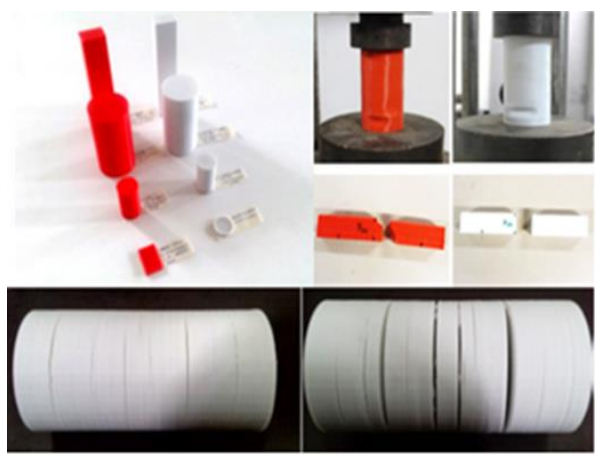

Figure 1: Evaluation of mechanical behavior of ABS and PLA. Martinez et al., 2019, p. 129-136.
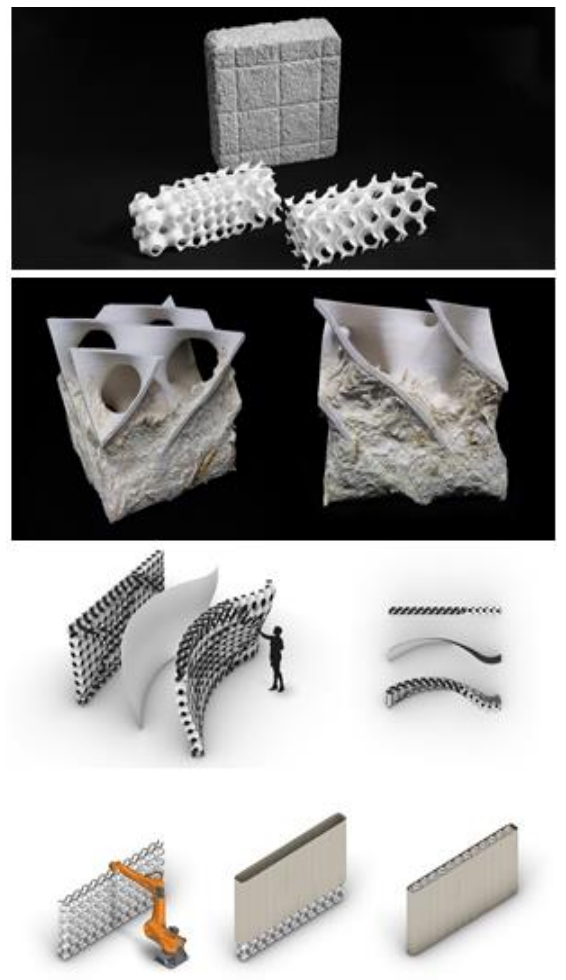

Figure 2: Mycelium composite in an adaptive lattice structure. Behforouz \& Pauletti, 2019.

\section{PERFORMANCE AND THE MATERIAL WORLD}

Digital design is part of a broader discussion about a supposed crisis in the fields of architectural theory and criticism, established since the 1990s (Sykes, 2010). According to this supposed crisis, theory has been superseded by practice in contemporaneity owing to the innocuous character of the former. It is no accident that this theoretical sterility coincides with the establishment of digital design practices. Suggesting that theory is being questioned nowadays, Sykes (2010) claims that, besides the opportunities and challenges of the digital domain, today's architectural discipline is evolving in response to the urgency of environmental issues and to an "attitude towards the 'real' (whatever the 'real' may be), that which exists and must be dealt with on practical and/or symbolic level" (Sykes, 2010, p.23).

As noted, the intertwining of the material and confronting environmental, ecological factors is already posed in digital design. Material-based design is now established as a paradigm of sustainable performance design subjugated to technological development. However, material-based design does not yet encompass, in theory or in practice, an in-depth understanding of the qualities of materials, which support the spheres of everyday human life and their multitude of external forces (historical, cultural, and political). In other words, it does not apprehend the connection between matter (and all of its associated processes) and the material world as do the material culture approach, studies on science and technology, and studies on technology and culture.

Studies of this nature call attention to concepts that deal with materials from a multidirectional perspective. They advocate the need for understanding materiality from the social point of view and the complex relationship between matter, material, objects, things and their constitutive processes.

Boivin (2010) highlights that the physical aspects of the material world, of materiality, shape our thoughts, emotions, and social relations, i.e., they shape human practice and thought. By calling for deeper integration of the human sciences and materials sciences, the author declares that not only does matter have action, but it also represents, and does not do so arbitrarily. However, the symbolic character of the agency of materials is complex, operating thoughts, emotions, and the very material dimension of human experiences.

Ingold (2012) highlights the material as a dynamic entity, as a 'matter flow' involved in transformation processes, which has no inert attributes. The author argues that it is necessary "to think from materials, not about them" (p.437), which helps to understand his argument that "materials are not in time; they are the stuff of time itself" (p.439). Ingold (2012) states that "materials are ineffable. They cannot be pinned down in terms of established concepts or categories" (p.435) and alerts to the limits of a vision that operates a distinction between the properties (scientifically measurable) and qualities (subjective) of materials. "To understand materials is to be able to tell their histories - of what they do and what happens to them when treated in particular ways - in the very practice of working with them. Materials do not exist as static entities with diagnostic 
attributes; [...]. Materials, thus, carry on, undergoing continual modulation as they do so. In the phenomena world, every material is a becoming" (Ingold, 2012, p.434435).

Tonuk (2018) shows that the materials come to be as they are because they result from iterative processes in relationships between several subjects and different disciplinary traditions, with their own history, cultural components, material practices, and infrastructures. However, materials are active agents, not 'passive victims' of external forces, including those who are involved in their making. Ingold (2012) highlights a "co-responsive movement of occurrent things along their manifold lines of becoming" (p.437).

Küchler (2015) focuses on the development of highperformance materials, emphasizes the need for a systems approach, "with continuous feedback from end-users and design specialists into the production and ongoing modification of materials" (p.275). According to this author, 'informed materials' result from screening and selecting suitable virtual candidates, whose properties are simulated, thereby reasserting materials as active design project elements that both resist and invite potentia transformation. However, in these scenarios, there are no more limitations to controlling the properties and performance of materials, and 'engineered materials' collapse the categories of artificial and natural; there is no control over their general social use in real life. This social implication cannot be predicted or imagined. In addition, Ihde and Malafouris (2019) stress the implications of relations between materiality and technical mediation: "Materiality and the forms of technical mediation that humans make and use are not passive or neutral but actively shape what we are in a given historical moment. The challenge for us is understanding in which ways and to what degree human beings are shaped and constituted by the stuff they make" (p.209)

As aforementioned, the material-based design paradigm disregards the qualities of matter, or of materials, except those related to their measurable attributes. From this perspective, the architect is responsible for crossdisciplinary collaboration, for establishing relations among the views on materials of chemists, physicists, and other scientists, for whom matter and materials are specific sets of attributes. However, architects risk not operating in depth the notion of tectonics, which evokes the potential for constructive expression of architecture, capable of linking material and structural aspects with cultural and aesthetic aspects (Frampton, 1985; Amaral, 2009). Kenneth Frampton's tectonic approach is, paradoxically, the same as that adopted and adapted by some digital design theorists, e.g., Oxman (2012).

Are we aware that material-based design operates a new material condition with historical, social, ethical, and political effects? Perhaps, but we have not yet incorporated these specific approaches that incorporate cultural and symbolic aspects, i.e., a more holistic view. Although nowadays we are subject to the inexorability of technical development and the seduction and enthusiasm for instrumentalization, we must keep technological determinism under control.

\section{TOWARDS MATERIAL-BALANCED DESIGN APPROACH}

As we have witnessed, there is a growing interest in matter(s), material substances, materiality, and material tectonics. In theoretical debates, the perspective is to operate properly the challenges that arise in practice. The adjustments and alternative approaches proposed in this study aim to help expand efforts so that the presently interdisciplinary practices may evolve towards transdisciplinarity. The architectural design centered on materiality, which incorporates sustainable performative perspectives, should also be able to encompass other dimensions of the constitution of the built environment.

Different from their Aristotelian-Thomistic ancestors, today's designers focus on the ontology of matter(s) or the process of material-based design, provided by analytical processes. While this focus has enabled considerable insight into performative features, it is rather sterile with regard to everyday and, in particular, scientific experiences of material symbolic aspects. In fact, most accounts leave epistemological problems and presuppositions undiscussed or implicit while agency plays a key role.

The technological horizons that lie ahead indicate that the tools and technical instruments will continue to assist us in design processes, allowing the creation of materiality consistent with needs and desires, balancing semantic and cultural issues with performative ones. The challenges of redirecting the training of designers are already given. We call attention to the maintenance of the essential consideration of apprehension of matter and materials (generally referred by us as materiality) in architecture beyond the limits and the imperative of optimum performances, which requires that we see all the possible implications of our options and decisions in establishing the world. The tools may not yet be ready to assist us in this task. The balance guiding us may derive from our capacity to integrate knowledge in a broad way. As discussed, we are imbricated in this horizon of technological development and the establishment of the material world.

This new concept of materiality can foster values, becoming an agent of social, ecological, and political equalities. We believe that it is possible to re-balance the way we inhabit our spaces, giving a higher value to the agency of the material, not only as a performative characteristic of the built environment but also as intent, promoting a common ground of principles.

Thus material-balanced design approach can address environmental, social, and cultural issues through a nove design praxis.

\section{REFERENCES}

ACADIA Association for computer aided design in architecture. (n.d.) Retrieved from http://acadia.org/events

Amaral, I. (2009) Quase tudo que você queria saber sobre tectônica, mas tinha vergonha de perguntar. Pós 16 (26), 148167

ASCAAD Arab society for computer aided architectural design. (n.d.) Retrieved from https://www.ascaad.org/activities.html

Bechthold, M. \& Weaver, J.C. (2017). Materials science and architecture. Nature Reviews Materials, 2, 1-20.

Behforouz K. \& Pauletti, I. Mycelium composite + adaptive lattice structure. Retrieved 
https://www.materialbalance.polimi.it/portfolio page/myceliu m-composite-adaptive-lattice-structure/

Boivin, N. (2010). Material cultures, material minds: The Impact of things on human thought, society, and evolution. New York: Cambridge University Press.

CAADRIA Association for computer-aided architectural design research in Asia. (n.d.) Retrieved from https://www.caadria.org/conf/past/

Caetano, I. \& Leitão, A. (2020). Architecture meets computation: An overview of the evolution of computational design approaches in architecture. Architectural Science Review, 63(2), 165-174. doi:doi.org/10.1080/00038628.2019.1680524

Carpo, M. (2017) The second digital turn: Design beyond intelligence. Cambridge, MA: The MIT Press.

Carpo, M. (Ed.) (2013) The digital turn in architecture 1992-2012 AD Reader. Chichester, UK: Wiley.

eCAADe Education and research in computer aided architectural design in Europe. (n.d.) Retrieved from https://ecaade.org/

Frampton, K. (1985) Modern architecture: A critical history. London: Thames and Hudson.

Gramazio, F. \& Kohler, M. (2008). Digital materiality in architecture. Baden: Lars Müller Publishers.

Gramazio, F., Kohler, M. \& Oesterle, S. (2010). Encoding material. Archit Design, 80: 108-115. doi:doi.org/10.1002/ad.1114

Inde, D. \&. Malafouris, L. (2019) Homo faber revisited: Postphenomenology and Material Engagement Theory. Philos. Technol. (32)195-214. doi:doi.org/10.1007/s13347018-0321-7

Ingold, T. (2012). Towards an ecology of materials. Annual Review of Anthropology, 41, 427-442.

Küchler, S. (2015) Materials: The story of use. In Drazin, A. \& Küchler, S. (Eds), The social life of materials: Studies in materials and society (pp. 267-282). London: Bloomsbury Academic.
Martinez, A. C. P., Souza, D. L., Santos, D. M., Pedroti, L. G., Carlo, J. C. \& Martins, M. A. D. (2019). Avaliação do comportamento mecânico dos polímeros ABS e PLA em impressão 3D visando simulação de desempenho estrutural. Gestão \& Tecnologia De Projetos, 14(1), 125-141. doi:doi.org/10.11606/gtp.v14i1.148289

Menges, A. (Ed.) (2012). Material computation: Higher integration in morphogenetic design. Architectural Design, 82 (2).

Oxman, N. (2010). Material-based design computation, PhD Thesis, Massachusetts Institute of Technology. Retrieved from https://dspace.mit.edu/handle/1721.1/59192

Oxman, R. \& Oxman, R. (2014). Theories of the digital in architecture. New York: Routledge.

Oxman, R. (2012). Informed tectonics in material-based design. Design Studies, 33(5), 427-455.

Oxman, R. (2017). Thinking difference: Theories and models of parametric design thinking. Design Studies, 52, 1-36.

SIGraDi Sociedad iberoamericana de gráfica digital. (n.d.) Retrieved from https://www.sigradi.org/

Sykes, A. K. (Ed.) (2010). Constructing a new agenda: Architectural theory 1993-2009. New York: Princeton Architectural Press.

Tonuk, D. (2018). Materials as temporally specific phenomena: Specialization and compromise in bioplastics production. Journal of Material Culture, 23(1), 114-130. doi:doi.org/10.1177/1359183517725547

Yazici, S. \& Tanacan, L. (2020). Material-based computational design (MCD) in sustainable architecture. Journal of Building Engineering, $32, \quad 101543 . \quad$ doi: doi.org/10.1016/j.jobe.2020.101543

Yazici, S., \& Tanacan, L. (2018). A study towards interdisciplinary research: $A$ material-based integrated computational design model (MICD-m) in architecture. Architectural Science Review, 61(1-2), 68-82. doi:doi.org/10.1080/00038628.2017.1416575 